\title{
Full Thickness Macular Hole Closure after Exchanging Silicone-Oil Tamponade with $\mathrm{C}_{3} \mathrm{~F}_{8}$ without Posturing
}

\author{
Tina Xirou ${ }^{a}$ Vasiliki Xirou $^{b}$ George Mangouritsas $^{a}$ \\ Elias Feretis $^{a}$ Stamatina A. Kabanarou ${ }^{a}$ \\ aRetina Department, Hellenic Red Cross General Hospital, Athens, Greece; \\ ${ }^{b}$ Department of Ophthalmology, Whipps Cross University Hospital, London, UK
}

\section{Key Words}

Internal limiting membrane peeling · Long-acting gas · Idiopathic macular hole ·

Vitrectomy · Silicone-oil tamponade

\begin{abstract}
Purpose: To report a case of macular hole closure after the exchange of a silicone-oil tamponade with gas $\mathrm{C}_{3} \mathrm{~F}_{8} 14 \%$.

Method: A 64-year-old female patient with a stage IV macular hole underwent a threeport pars-plana vitrectomy and internal limiting membrane peeling. Due to the patient's chronic illness (respiratory problems), a silicone-oil tamponade was preferred. However, the macula hole was still flat opened four months postoperatively. Therefore, the patient underwent an exchange of silicone oil with gas $C_{3} F_{8} 14 \%$. No face-down position was advised postoperatively due to her health problems.
\end{abstract}

Results: Macular hole closure was confirmed with optical coherence tomography six weeks after exchanging the silicone oil with gas.

Conclusions: Macular hole surgery using a silicone-oil tamponade has been proposed as treatment of choice for patients unable to posture. In our case, the use of a long-acting gas $\left(C_{3} F_{8} 14 \%\right)$, even without posturing, proved to be more effective.

\section{Introduction}

Idiopathic macular hole is a common cause of visual impairment in people in their sixth decade or older, with a higher prevalence in females [1]. Pars-plana vitrectomy was introduced as treatment of choice for a full thickness macula hole (FTMH) in the early 1990s [2]. Since then, a number of modifications in the surgical technique have been 
introduced in order to achieve anatomical restoration of the macular area. Internal limiting membrane (ILM) peeling [3] and the use of substances such as autologous platelets and/or serum have been proposed as adjuncts to the standard procedure to achieve a higher success rate. The use of a gas tamponade and face-down position postoperatively were considered mandatory for optimal results.

We report a case of a FTMH (stage IV), which was initially treated with pars-plana vitrectomy, ILM peeling and a silicone-oil tamponade. Anatomical closure was achieved only when the silicone oil was replaced by long-acting gas $\mathrm{C}_{3} \mathrm{~F}_{8}(14 \%)$, four months after the first surgery.

\section{Case Report}

A 64-year-old female patient was referred to our clinic with complaints of a gradual deterioration of vision in her left eye over the last six months. On initial examination, Snellen visual acuity was 0.9 in her right eye and 0.1 in her left eye. Slit-lamp biomicroscopy revealed no pathology in the anterior segment. Dilated fundus examination showed a normal fundus in her right eye, but revealed a macular hole in her left eye. Optical coherence tomography (OCT) confirmed the presence of a stage IV macular hole (fig. 1). The patient had undergone an uneventful cataract surgery with intraocular lens implantation in both eyes the previous year.

The patient underwent a standard vitrectomy procedure followed by air-fluid exchange and ILM peeling using membrane blue. Silicone oil (1,000 centistokes) was used as the optimal tamponade instead of a long-acting gas, as postoperative posturing was not possible for the patient due to her respiratory problems. No intra- or postoperative complications occurred. Four months later, the visual acuity in her left eye was still 0.1, and OCT imaging showed that the macular hole was still flat open (fig. 2). The patient underwent a second surgical procedure where the silicone oil was exchanged with a long-acting gas, $\mathrm{C}_{3} \mathrm{~F}_{8}(14 \%)$, without any further intervention or posturing. Macular hole closure was confirmed by OCT six weeks later and has remained stable since then (ig. 3 ). Visual acuity was improved to 0.3 one year postoperatively.

\section{Discussion}

Following successful pars-plana vitrectomy for FTMH, glial and Müller cells form a plug within the hole and this allows the edges to close. The presence of residual fluid in the hole postoperatively may split the plug formation and inhibit the approximation of the hole edges [4]. The use of a tamponade provides a floatation force that maintains the macula dry and keeps the edges in close proximity.

Long-acting gas remains the tamponade of choice, providing sufficient floatation force, which is greater in a face-down position [4]. Furthermore, in comparison to the use of silicone oil, which needs removal in a second surgical intervention, a subsequent procedure is not required. However, silicone oil is still an alternative option for patients with specific health problems (e.g. in patients who are not able to posture) and in patients with a single eye who require rapid rehabilitation. Silicone-oil tamponade has also been proposed for the management of large or re-opened macular holes [5]. It has been reported that prolonged macular tamponade with long-acting gas and a strict face-down position has a high closure rate, even in holes larger than $400 \mu \mathrm{m}[4,6]$. On the contrary, Tornambe et al. [7] described good anatomical results with the use of long-acting gas regardless of the head position. 
The use of silicone oil in FTMH surgery has been shown to be effective, with a high anatomical success rate but contradictory functional outcome $[5,8,9]$. In our case, not only silicone-oil tamponade did not lead to anatomical restoration but the macular hole healed without posturing with the use of long-acting gas $\mathrm{C}_{3} \mathrm{~F}_{8}(14 \%)$.

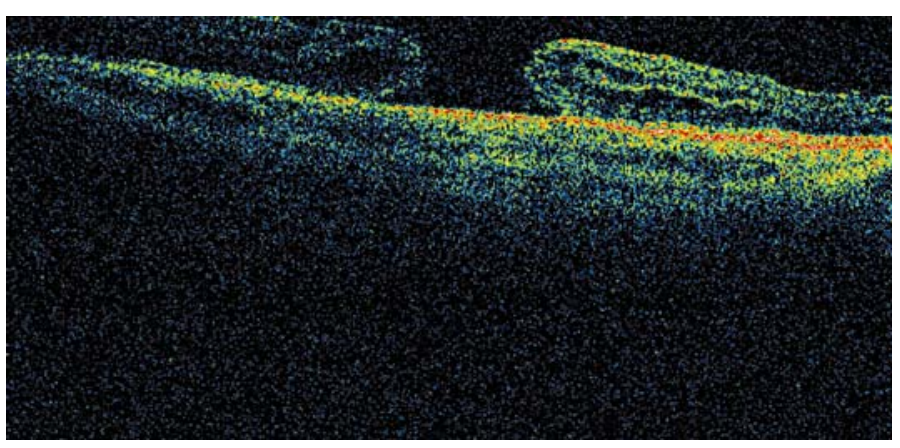

Fig. 1. OCT examination shows a stage IV macular hole with intraretinal fluid.

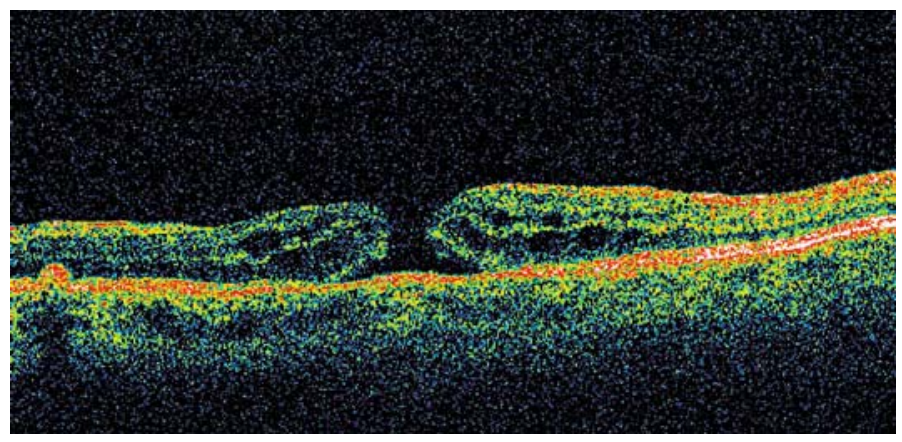

Fig. 2. OCT examination reveals a flat open hole. The highly reflective surface corresponds to the silicone oil that fills the vitreous cavity. 


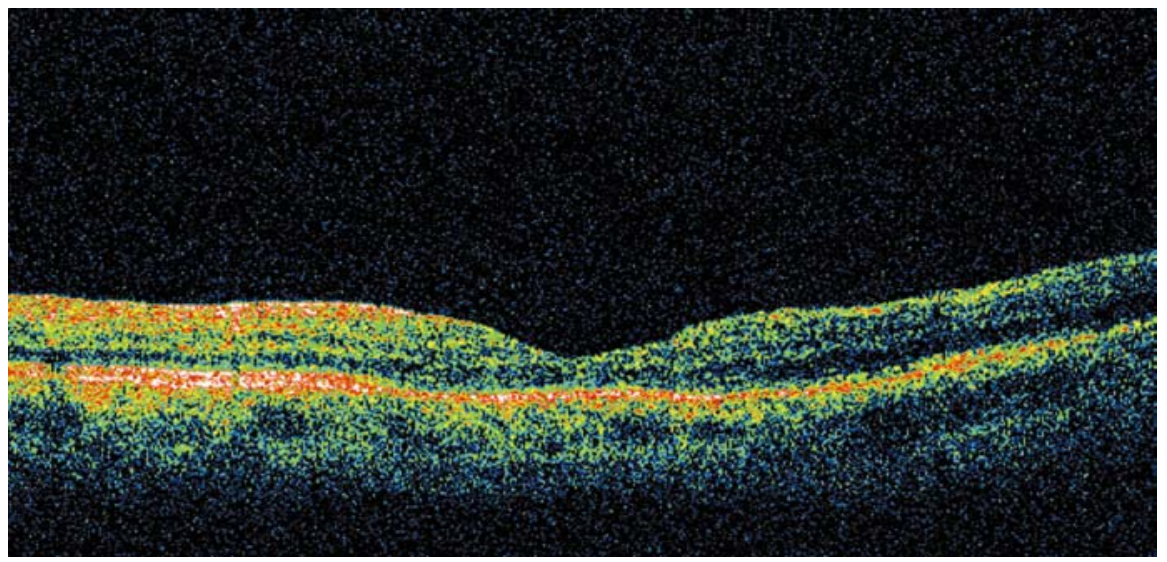

Fig. 3. OCT imaging showing macular hole closure after the second surgical procedure and the anatomical restoration of the foveal contour.

\section{References}

1 Gass JDM: Risk of developing a macular hole. Arch Ophthalmol 1991;109:611.

2 Kelly NE, Wendel RT: Vitreous surgery for idiopathic macular holes. Results of a pilot study. Arch Ophthalmol 1991;109:654-659.

-3 Brooks HL Jr: Macular hole surgery with and without internal limiting membrane peeling. Ophthalmology 2000;107:1939-1949.

-4 Thompson JT, Smiddy WE, Glaser BM, Sjaarda RN, Flynn HW Jr: Intraocular tamponade duration and success of macular hole surgery. Retina 1996;16:373-382.

5 Pertile G, Claes C: Silicone oil versus gas for the treatment of full-thickness macular hole. Bull Soc Belge Opthalmol 1999;274:31-36.

6 Guillaubey A, Malvitte L, Lafontaine PO, Jay N, Hubert I, Bron A, Berrod JP, Creuzot-Garcher C: Comparison of face-down position after idiopathic macular hole surgery: a randomised clinical trial. Am J Ophthalmol 2008;146:128-134.

7 Tornambe PE, Poliner LS, Grote K: Macular hole surgery without face-down positioning. A pilot study. Retina 1997;17:179-185.

8 Karia N, Laidlaw A, West J, Ezra E, Gregor MZ: Macular hole surgery using silicone oil tamponade. Br J Ophthalmol 2001;85:1320-1323.

-9 Goldbaum MH, McCuen BW, Hanneken AM, Burgess SK, Chen HH: Silicone oil tamponade to seal macular holes without position restrictions. Ophthalmology 1998;105:2140-2147.

The authors have no proprietary interest in the presented material, and there was no financial support for this study. 